Provided for non-commercial research and education use. Not for reproduction, distribution or commercial use.

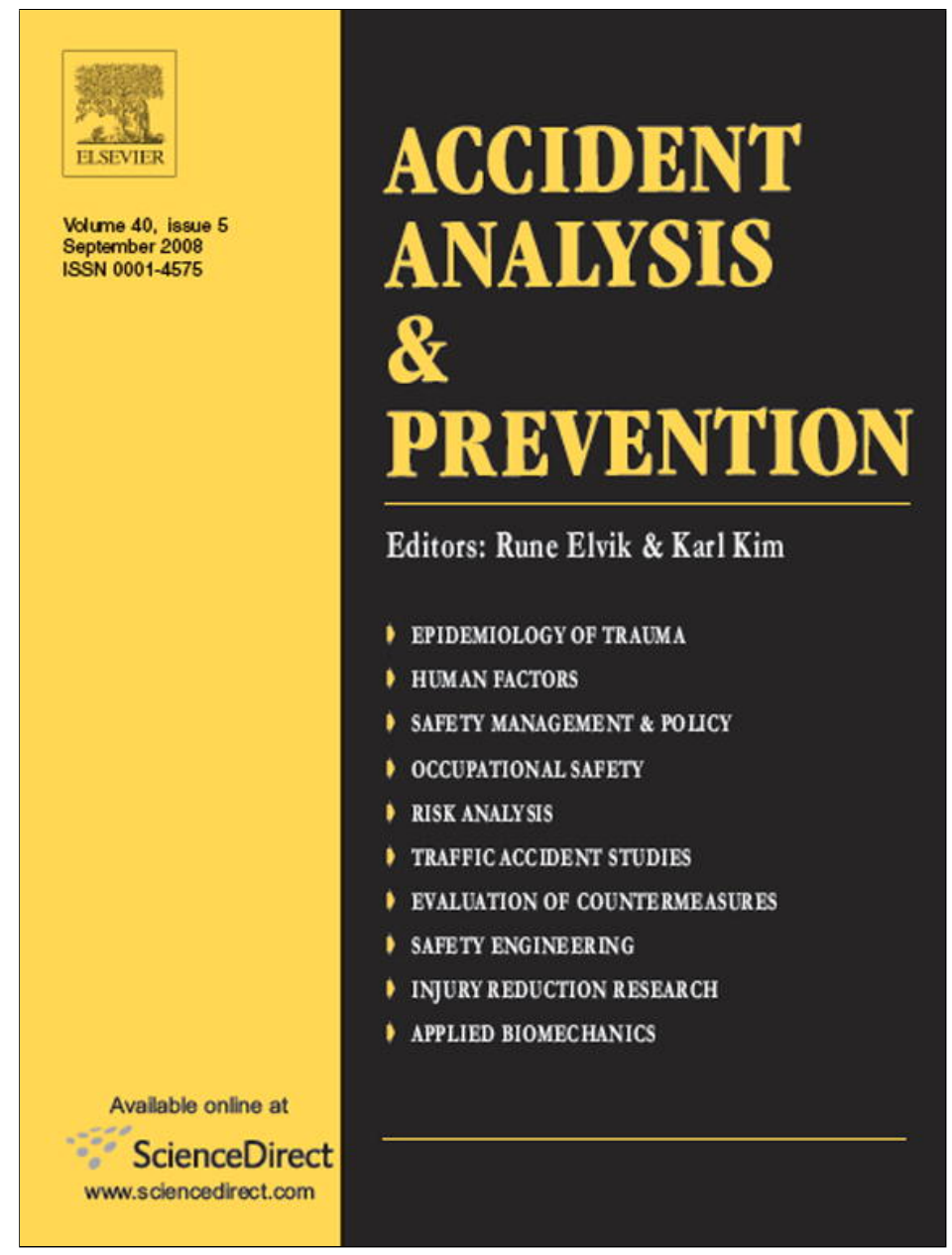

This article appeared in a journal published by Elsevier. The attached copy is furnished to the author for internal non-commercial research and education use, including for instruction at the authors institution and sharing with colleagues.

Other uses, including reproduction and distribution, or selling or licensing copies, or posting to personal, institutional or third party websites are prohibited.

In most cases authors are permitted to post their version of the article (e.g. in Word or Tex form) to their personal website or institutional repository. Authors requiring further information regarding Elsevier's archiving and manuscript policies are encouraged to visit:

http://www.elsevier.com/copyright 


\title{
Development of crash-severity-index models for the measurement of work zone risk levels
}

\author{
Yingfeng $\mathrm{Li}^{\mathrm{a}, \mathrm{c}, 1}$, Yong Bai ${ }^{\mathrm{b}, *}$ \\ a 1530 W. 15th Street, 2160 Learned Hall, Department of Civil, Environmental, and Architectural Engineering, The University of Kansas, Lawrence, KS 66045, USA \\ b 1530 W. 15th Street, 2135-B Learned Hall, Department of Civil, Environmental, and Architectural Engineering, The University of Kansas, Lawrence, KS 66045, USA \\ c Texas Transportation Institute, San Antonio, TX 78229, USA
}

\section{A R T I C L E I N F O}

\section{Article history:}

Received 12 October 2007

Received in revised form 23 May 2008

Accepted 5 June 2008

\section{Keywords:}

Crash

Highway

Risk

Safety

Work zone

\begin{abstract}
A B S T R A C T
Highway work zones interrupt regular traffic flows and create safety problems. Improving safety without sacrificing the main function of highways is a challenging task that traffic engineers and researchers have to confront. In this study, the concept of using crash severity index (CSI) for work zone safety evaluation was proposed and a set of CSI models were developed through the modeling of work zone crash severity outcomes. A CSI is a numerical value between zero and one that is estimated from given work zone variables. It is interpreted as the likelihood of having fatality/fatalities when a severe crash occurs in a given work zone. The CSI models were developed using a three-step approach. First, a wide range of crash variables were examined in a comprehensive manner and the significant risk factors that had impact on crash severity were selected. Second, the CSI models were developed using logistic regression technique by incorporating the selected risk factors. Finally, the developed models were validated using the recent crash data and their ability in assessing work zone risk levels were analyzed. Results of this study showed that CSI models can provide straightforward measurements of work zone risk levels.
\end{abstract}

(C) 2008 Elsevier Ltd. All rights reserved.

\section{Introduction}

As the highway system ages, government agencies have to allocate a greater percentage of their funding on preserving, expanding, and enhancing existing highway networks. Work zones on the highway system interrupt regular traffic flows and create safety problems. Improving safety without sacrificing the main function of highways has become a challenging task that traffic engineers and researchers have to confront.

Work zone safety can be affected by combinations of various risk factors and some combined effects might not be fully recognized during work zone designs. Understanding risks discovered from work zone crash data analyses is a key step towards lowering risk levels and preventing the occurrence of severe crashes. In this study, the concept of the crash severity index (CSI) was proposed for the evaluation of risk levels in work zones. A CSI is designed to be a numerical value between zero and one that can be estimated from given work zone risk factors. It is interpreted as the likelihood of having fatality/fatalities when a severe crash occurs in a given work zone. When quoted hereafter, severe crashes refer to crashes involv-

\footnotetext{
* Corresponding author. Tel.: +1 785864 2991; fax: +1 7858645631.

E-mail addresses: y-li@tamu.edu (Y. Li), ybai@ku.edu (Y. Bai).

1 Tel.: +1 210731 9938x34; fax: +1 2107318904
}

ing fatality/fatalities (i.e., fatal crashes) or injury/injuries (i.e., injury crashes) of either passengers or drivers of the involved vehicles. In this study, the CSI models were developed through the modeling of work zone crash severity outcomes based on the work zone fatal and injury crash data in Kansas.

A CSI reflects the risk level of a given work zone assuming that the work zone will have a high risk level for travelers if the likelihood of having fatality/fatalities in a severe crash is high. To develop the CSI models, chi-square statistics and Cochran-Mantel-Haenszel (CMH) statistics were first utilized to identify the significant risk factors. The logistic regression method was then deployed to develop the models. CSI models provide straightforward measurements of work zone risk levels based on a wide range of variables that may contribute to severe crashes. Traffic engineers can use the developed models to assess the risk level for either an existing work zone or a newly proposed work zone, which provides an opportunity to develop safety countermeasures to eliminate or mitigate the risks for the traveling public.

\section{Literature review}

The logistic regression technique was selected for the CSI model development in this study. Logistic regression models are direct probability models that have no requirements on the distributions 
of the explanatory variables or predictors (Harrell, 2001). This technique is more flexible and more likely to yield accurate results in traffic crash analyses where the safety impact of contributing factors needs to be quantified. In addition, logistic regression models generate outcome values between zero and one, which makes this statistical method ideal for developing models to estimate numerical outcomes with specified ranges.

The significance of logistic regression technique in the analysis of traffic safety has been recognized for years. Hill (2003) and Li and Bai (2006) utilized this technique in the analysis of work zone fatal crashes to quantify the effectiveness of traffic control devices. The technique was also used to model the relationships between crashes severity and wide ranges of crash variables. Lu et al. (2006) utilized logistic regression to develop models to predict the severity of median crossover crashes in Wisconsin. Chang and Yeh (2006) used the logistic regression in their analysis of fatality risk factors for motorcyclists in Taiwan. The logistic regression was deployed by Kim et al. (2000) in their analyses of alcohol impact on motorcycle crashes. In their analyses, a logistic regression model was developed to explain the likelihood of an alcohol-related motorcycle crash as a function of rider characteristics and environmental and temporal factors.

Other similar methods were also used in previous crash severity analyses. Dissanayake and Lu (2002) developed a set of sequential binary logistic regression models to analyze the contributing factors and predict the crash severity of single-vehicle fixedobject crashes involving young drivers. The researchers utilized the SAS software package to develop the regression models that took into account crash factors such as gender, driver impairment, and geometric conditions of crash locations. Ouyang et al. (2002) developed a simultaneous binary logit model to address the relationships between injury severity outcomes and various crash factors involved in car-truck collisions.

In summary, literature search showed that the logistic regression has been applied to several crash severity analyses, as briefly reviewed above. However, the relationships between crash severities and multiple risk factors in highway work zones have not been fully explored. The concept of using CSI to evaluate the driving risk levels in existing or proposed highway work zones was not found in previous publications either.

\section{Data description}

The crash data used for CSI model development contained 85 fatal crashes between 1998 and 2004, and 604 injury crashes between 2003 and 2004 in Kansas highway work zones. The crash data were originally obtained from the Kansas Department of Transportation (KDOT) database. The KDOT database included three levels of crash severity including fatal (i.e., crashes involved fatality/fatalities), injury (crashes involved injury/injuries only), and property-damage-only (crashes without injury or fatalities). For this study, only fatal and injury crashes were analyzed. The original format of the data was that a single crash was frequently described in text in multiple data rows because of multiple vehicles, traffic control devices, or contributing factors involved. This data format could not be directly utilized for computer-aided analyses using software such as SAS. Thus, the format of crash data has to be changed using the following two steps. First, at-fault drivers were identified and their characteristics were compiled along with other crash information into spreadsheets where each crash was described in a single data row. Then, for the cases with missing or unclear information, the original crash reports, including detailed crash scene descriptions and sketches, were examined to ensure the data accuracy.
The collected crash information was organized into five categories. Each category included various crash variables with specific observations. Each observation was assigned with a number, as shown in Table 1. Some observations were combined to form more general observation groups so that the frequencies of the cross-categorized observations were increased. The increased data frequencies would minimize the errors caused by data sparseness in statistical tests and logistic regression. Some major traffic control methods and dominant driver errors associated with the crashes were also included as crash variables and their values were shown in Table 2.

\section{Development of work zone crash-severity-index models}

A set of CSI models were developed based on the information of severe work zone crashes involving injuries and fatalities. The procedure of model development included three steps. First, the risk factors in work zones that had impact on crash severity were determined based on the collected crash data. Second, a set of CSI models were developed by incorporating these risk factors using the logistic regression technique. Finally, the predictability of the developed models was validated using the most recent work zone crash data.

The collected crash data were divided into two groups. The dataset used for risk factor determination and model development had a total of 334 severe work zone crashes including 67 fatal crashes between 1998 and 2003 and 267 injury crashes in 2003. Adding the additional fatal crashes (1998-2002) in the model development dataset enriched the fatal crash information and thus increased model accuracy, especially for estimating CSIs at high risk level (i.e., a risk level at which fatal crashes may occur). The dataset for model verification included 355 severe crashes in year 2004 in Kansas highway work zones, among which 18 were fatal crashes and 337 were injury crashes.

\subsection{Work zone risk factor determination}

The determination of risk factors associated with work zone crash severity was a critical step towards developing CSI models with high accuracy and predictability. The determination process involved an examination of 29 work zone crash variables. Some of the variables may have negligible impact on the crash severity. These variables should be abandoned because incorporating them in the CSI models might not only complicate the models, but also lower their accuracies. Although most of the crash variables were mutually independent, some variables were associated with others and certain combinations of these variable pairs may interactively affect the crash severity. Thus, identifying the risk factors that both individually and interactively affect work zone crash severity became critical.

Chi-square statistics and Cochran-Mantel-Haenszel (CMH) statistics were employed to ensure the accuracy of risk factor identification. As shown in Fig. 1, the identification procedure included the following three steps and through which 18 out of 29 variables were selected as risk factors as listed in Table 3.

Step 1 The variables that are statistically associated with the crash severity were selected first as risk factors through chi-square statistics. Pearson chi-square and likelihood ratio chi-square tests were utilized in this step. A variable was selected when at least one of the two tests supported its relationship with the crash severity (i.e., a $p$-value less than or equal to the 0.1 level of significance). 
Table 1

Data categories and variables

\begin{tabular}{|c|c|c|c|}
\hline Category & Variable & Observation & Assigned value \\
\hline \multirow[t]{9}{*}{ Driver at fault ${ }^{\mathrm{a}}$} & \multirow[t]{7}{*}{ Age } & $15-19$ & 1 \\
\hline & & $20-24$ & 2 \\
\hline & & $25-34$ & 3 \\
\hline & & $35-44$ & 4 \\
\hline & & $45-54$ & 5 \\
\hline & & $55-64$ & 6 \\
\hline & & $\geq 65$ & 7 \\
\hline & \multirow[t]{2}{*}{ Gender } & Male & 1 \\
\hline & & Female & 2 \\
\hline \multirow[t]{11}{*}{ Time } & \multirow[t]{4}{*}{ Time of day (h) } & $6: 00-10: 00$ & 1 \\
\hline & & 10:00-16:00 & 2 \\
\hline & & $16: 00-20: 00$ & 3 \\
\hline & & 20:00-6:00 & 4 \\
\hline & \multirow[t]{7}{*}{ Day of week } & Monday & 1 \\
\hline & & Tuesday & 2 \\
\hline & & Wednesday & 3 \\
\hline & & Thursday & 4 \\
\hline & & Friday & 5 \\
\hline & & Saturday & 6 \\
\hline & & Sunday & 7 \\
\hline \multirow[t]{9}{*}{ Environmental conditions } & \multirow[t]{4}{*}{ Light condition } & Good condition i.e., daylight & 1 \\
\hline & & Fair conditions including dawn, dusk, and dark with streetlights & 2 \\
\hline & & Poor condition i.e., dark without streetlights & 3 \\
\hline & & Other unfavorable light conditions & 4 \\
\hline & \multirow[t]{2}{*}{ Weather condition } & Good condition i.e., no adverse conditions & 1 \\
\hline & & $\begin{array}{l}\text { Poor conditions including rain, mist, drizzle, sleet, snow, fog, smoke, strong winds, blowing } \\
\text { dust or sand, freezing rain, rain and fog, rain and wind, sleet and fog, snow and winds, and } \\
\text { other }\end{array}$ & 2 \\
\hline & \multirow[t]{3}{*}{ Road surface condition } & Good condition i.e., dry surface & 1 \\
\hline & & Fair conditions including wet, mud, dirt, sand, and debris & 2 \\
\hline & & Poor conditions including snow, slush, ice, and snow packed & 3 \\
\hline \multirow[t]{23}{*}{ Road conditions } & \multirow[t]{3}{*}{ Road class } & Interstates and other freeways and expressways & 1 \\
\hline & & Other principal arterials and minor arterials & 2 \\
\hline & & Low-classification roads including major collectors, minor collectors, and local roads & 3 \\
\hline & \multirow[t]{5}{*}{ Road character } & Straight and level & 1 \\
\hline & & Straight on grade & 2 \\
\hline & & Curve and level & 3 \\
\hline & & Curve on grade & 4 \\
\hline & & Other geometric alignments & 5 \\
\hline & Number of lanes & Actual number of the traffic lanes in two directions & - \\
\hline & \multirow[t]{4}{*}{ Speed limit (mph) } & $\geq 61$ & 1 \\
\hline & & $51-60$ & 2 \\
\hline & & $41-50$ & 3 \\
\hline & & $\leq 40$ & 4 \\
\hline & \multirow[t]{3}{*}{ Crash location } & Non-intersection areas & 1 \\
\hline & & Intersection or Intersection related areas & 2 \\
\hline & & Other areas including interchange areas, crossover areas, and other & 3 \\
\hline & \multirow[t]{3}{*}{ Surface type } & Concrete & 1 \\
\hline & & Blacktop & 2 \\
\hline & & Other & 3 \\
\hline & \multirow[t]{2}{*}{ Road special feature } & No special feature impact & 0 \\
\hline & & $\begin{array}{l}\text { Impacted by special features including bridge, overhead bridge, railroad bridge, railroad } \\
\text { crossing, interchange, ramp, and other }\end{array}$ & 1 \\
\hline & \multirow[t]{2}{*}{ Area information } & Urban area & 1 \\
\hline & & Rural area & 2 \\
\hline \multirow[t]{3}{*}{ Crash information } & Vehicle body type & Truck $^{\mathrm{b}}$ involved & 1 \\
\hline & & Non-truck involved & 2 \\
\hline & No. of vehicles & Actual number of the vehicles involved in a crash & - \\
\hline
\end{tabular}

a Driver at fault was the person who caused a crash according to an accident report. For a single-vehicle crash case, the driver of the crash vehicle was automatically considered as the driver at fault.

b Trucks include single large trucks, truck and trailers, tractor-trailers, and buses.

Step 2 The insignificant variables from the previous step were further examined by CMH statistics at 0.1 level of significance to detect those that affect work zone crash severity interactively with certain selected risk factors. The direct impact of these variables may not strong enough to be statistically detected through chi-square tests. $\mathrm{CMH}$ statistics test the relationships between initially unselected variables and the crash severity variable in a three-way contingency table by controlling the selected risk factors. Some previous applications of $\mathrm{CMH}$ statistics in crash data exploration can be found in Chirsa-Chavala and Mak (1986) and Chen and Jovanis (2000). The significant variables supported by CMH statistics in this step were selected as risk factors. The $\mathrm{CMH}$ statistics used in this study included the nonzero correlation statistic, the row mean scores statistic, and the general association statistic. 
Table 2

Traffic control and driver error variables

\begin{tabular}{lll}
\hline Category & Variable & Variable values \\
\hline Traffic control & None or inoperative & 0 (not present); 1 (present) \\
& Officer or flagger & 0 (not present); 1 (present) \\
& Stop sign/signal & 0 (not present); 1 (present) \\
& Flasher & 0 (not present); 1 (present) \\
& No-passing zone & 0 (not present); 1 (present) \\
& Center/edge lines & 0 (not present); 1 (present) \\
Driver error & No driver error & 0 (not present); 1 (present) \\
& Drug or alcohol impairment & 0 (not present); 1 (present) \\
& Disregarded traffic signs, signals, & 0 (not present); 1 (present) \\
& and markings & \\
& Exceeded posted speed limits or & 0 (not present); 1 (present) \\
& too fast for conditions & \\
& Following too closely & 0 (not present); 1 (present) \\
& Inattentive drivinga & 0 (not present); 1 (present)
\end{tabular}

a Inattentive driving includes such errors on the KDOT accident reports as "fell asleep," "inattention," "other distraction in or on vehicle," "distraction-cell phone," and "distraction-other electronic devices."

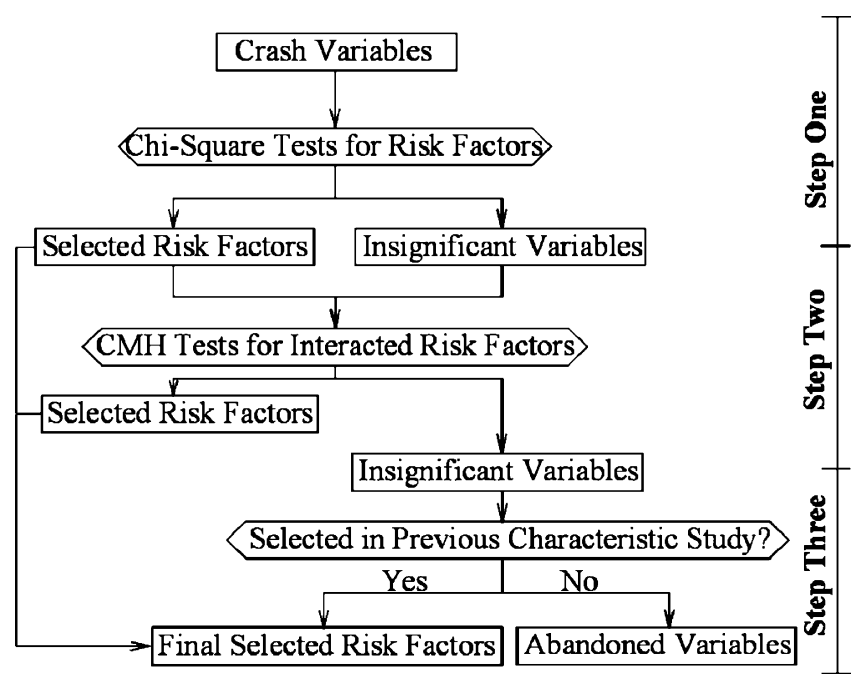

Fig. 1. Risk factor selection flowchart.

Table 3

Selected work zone risk factors

\begin{tabular}{rlll}
\hline No. & Risk factor & Abbr. & Selection step \\
\hline 1 & Age & AG & First step \\
2 & Light condition & LC & First step \\
3 & Vehicle type & VT & First step \\
4 & Road class & RC & First step \\
5 & Road character & RCH & First step \\
6 & Number of lanes & LN & First step \\
7 & Speed limit & SL & First step \\
8 & Surface type & SUR & First step \\
9 & None/inoperative traffic control & NTC & First step \\
10 & Flagger & FL & First step \\
11 & Stop sign/signal & ST & First step \\
12 & Disregarded traffic control & DTC & First step \\
13 & Following too close & FC & First step \\
14 & Crash time & CT & Second step \\
15 & Special feature & SF & Second step \\
16 & Area information & AI & Second step \\
17 & Alcohol/drug impairment & AL & Third step \\
18 & Exceeded posted speed limits or too & SP & Third step \\
& fast for conditions & & \\
\hline
\end{tabular}

Step 3 To identify all potential risk factors, the results of the characteristic comparisons between fatal and injury crashes were examined. Characteristic comparisons between fatal and injury were conducted in a previous project by authors and some of the results were utilized for this study directly. Risk factors that were identified based on the previous comparison study yet not detected in the steps 1 and 2 were also selected. As unveiled in the previous comparison study, factors such as alcohol/drug impairment and too fast for conditions/speeding had significant impact on crash severity outcomes but were not selected in the first two steps ( $\mathrm{Li}$, 2007).

\subsection{Development of CSI models}

Based on the selected risk factors, two groups of CSI models were developed using logistic regression including two driver-independent CSI (DI-CSI) models as one group and two driver-dependent CSI (DD-CSI) models as the other group. The DICSI models only included the risk factors that described the travel conditions in highway work zones. These models can be used to estimate the driving risks in work zones without knowing human factors. The estimated CSI values reflect the risk levels of proposed or existing highway work zones for traveling public. The DD-CSI models, on the other hand, are associated with particular drivers by including not only the risk factors related to work zones but also those risk factors that only certain drivers may possess such as demographic characteristics and driver errors.

\subsubsection{Developed DI-CSI models}

A DI-CSI model, or the comprehensive DI-CSI model, was first generated using SAS which included all driver-independent risk factors, as listed in Eq. (1). Table 4 lists the estimated variable coefficients and related statistical results for the comprehensive DI-CSI model. The Wald chi-square statistic was used to test the variable significance for the logistic regression models. SAS also outputted the values of three statistics for assessing the goodness-of-fit for the logistic regression model including the AIC statistic, the SC statistic, and the $-2 \log$ likelihood statistic. The log likelihood statistic was used to test the global null hypothesis that all the parameters associated with covariates were zero (under the null hypothesis, the -2 log likelihood statistic has a chi-square distribution). The AIC (Akaike information criterion) and SC (Schwarz criterion) statistics adjusted the $-2 \log$ likelihood statistic for the number of terms

Table 4

Variables and coefficients for the comprehensive DI-CSI model

\begin{tabular}{lrlcr}
\hline Variable & Coeff. & Standard error & Wald chi-square & $p$-Value \\
\hline Constant & 7.62 & 2.20 & 12.00 & 0.001 \\
Crash time (CT) & -0.11 & 0.22 & 0.26 & 0.613 \\
Light condition (LC) & 0.55 & 0.29 & 3.46 & 0.063 \\
Vehicle type (VT) & -0.91 & 0.36 & 6.19 & 0.013 \\
Road class (RC) & -0.67 & 0.53 & 1.57 & 0.210 \\
Road character (RCH) & 0.13 & 0.15 & 0.74 & 0.389 \\
No. of lanes (LN) & -0.86 & 0.23 & 13.61 & $<0.001$ \\
Speed limit (SL) & -0.74 & 0.23 & 10.36 & 0.001 \\
Surface type (SUR) & 0.29 & 0.41 & 0.48 & 0.490 \\
Special feature (SF) & -0.59 & 0.48 & 1.52 & 0.218 \\
Area information (AI) & -1.74 & 0.61 & 8.05 & 0.005 \\
None/inoperative traffic & -2.69 & 1.09 & 6.04 & 0.014 \\
control (NTC) & & & & 0.427 \\
Flagger (FL) & -0.48 & 0.60 & 0.63 & 0.021 \\
Stop sign/signal (ST) & 1.51 & 0.66 & 5.31 &
\end{tabular}

$\mathrm{AIC}=258.8 ; \mathrm{SC}=312.1 ;-2 \log$ likelihood $=230.8$. Testing global null hypothesis: $\beta=0$ : likelihood ratio chi-square (chi-square value, $p$-value): $104.1,<0.001$; score chi-square (chi-square value, $p$-value): $89.6,<0.001$; Wald chi-square (chi-square value, $p$-value): $58.3,<0.001$. 
Table 5

Variables and coefficients for the simplified DI-CSI model

\begin{tabular}{lrlcr}
\hline Variable & Coeff. & Standard error & Wald chi-square & $p$-Value \\
\hline Constant & 7.64 & 2.06 & 13.79 & $<0.001$ \\
Light condition (LC) & 0.54 & 0.20 & 7.40 & 0.007 \\
Vehicle type (VT) & -0.93 & 0.36 & 6.67 & 0.010 \\
Road class (RC) & -0.59 & 0.52 & 1.27 & 0.260 \\
Special feature (SF) & -0.54 & 0.45 & 1.43 & 0.232 \\
No. of lanes (LN) & -0.86 & 0.23 & 14.16 & $<0.001$ \\
Speed limit (SL) & -0.70 & 0.22 & 9.79 & 0.002 \\
Area information (AI) & -1.62 & 0.60 & 7.25 & 0.007 \\
Non/inoperative traffic & -2.71 & 1.09 & 6.21 & 0.013 \\
control (NTC) & & & & \\
Stop sign/signal (ST) & 1.40 & 0.64 & 4.78 & 0.029
\end{tabular}

$\mathrm{AIC}=252.9 ; \mathrm{SC}=291.0 ;-2 \log$ likelihood $=232.9$. Testing global null hypothesis: $\beta=0$ likelihood ratio chi-square (chi-square value, $p$-value): $101.9,<0.001$; score chisquare (chi-square value, $p$-value): $88.4,<0.001$; Wald chi-square (chi-square value, p-value): $57.8,<0.001$

in the model and the number of observations used. These statistics are used when comparing different models for the same data and lower values of these statistics indicate a model with better goodness-of-fit (SAS, 2003):

comprehensive DI - CSI model : DI - CSI $=\frac{\exp \left[g_{1}(\boldsymbol{x})\right]}{1+\exp \left[g_{1}(\boldsymbol{x})\right]}$

where $\quad g_{1}(\boldsymbol{x})=7.62-0.11 \mathrm{CT}+0.55 \mathrm{LC}-0.91 \mathrm{VT}-0.67 \mathrm{RC}+0.13 \mathrm{RCH}$ $-0.86 \mathrm{LN}-0.74 \mathrm{SL}+0.29 \mathrm{SUR}-0.59 \mathrm{SF}-1.74 \mathrm{AI}-2.69 \mathrm{NTC}-0.48 \mathrm{FL}$

$+1.51 \mathrm{ST}$ and the descriptions of the variables can be found in Table 1.

In Table 4, the $p$-values of some variables, such as crash time, road character, surface type, and flagger/officer, are large (i.e., larger than the pre-set criterion of 0.3). From the statistical viewpoint, dropping these variables from the regression model does not lose much data information. Thus, a simplified DI-CSI model (Eq. (2)) was developed by including only the statistically significant variables that had relatively small $p$-values. The variables coefficients of the second DI-CSI model are presented in Table 5:

Simplified DI - CSI model : DI - CSI $=\frac{\exp \left[g_{2}(\boldsymbol{x})\right]}{1+\exp \left[g_{2}(\boldsymbol{x})\right]}$

where $\quad g_{2}(\boldsymbol{x})=7.64+0.54 \mathrm{LC}-0.93 \mathrm{VT}-0.59 \mathrm{RC}-0.54 \mathrm{SF}-0.86 \mathrm{LN}$ $-0.70 \mathrm{SL}-1.62 \mathrm{AI}-2.71 \mathrm{NTC}+1.40 \mathrm{ST}$.

\subsubsection{Developed DD-CSI models}

A pair of DD-CSI models was also developed by considering both work zone variables and driver characteristics. The comprehensive DD-DSI model generated by SAS was presented in Eq. (3). This model included all risk factors that were selected from the candidate crash variables. Table 6 lists the estimated variable coefficients for the model:

comprehensive DD - CSI model : DD $-\mathrm{CSI}=\frac{\exp \left[g_{3}(\boldsymbol{x})\right]}{1+\exp \left[g_{3}(\boldsymbol{x})\right]}$

where $g_{3}(\boldsymbol{x})=5.25+0.03 \mathrm{CT}+0.51 \mathrm{LC}-0.80 \mathrm{VT}-0.59 \mathrm{RC}+0.16 \mathrm{RCH}$ $-0.70 \mathrm{LN}-0.84 \mathrm{SL}+0.40 \mathrm{SUR}-0.37 \mathrm{SF}-1.69 \mathrm{AI}-2.52 \mathrm{NTC}-0.82 \mathrm{FL}$ $+0.78 \mathrm{ST}+0.32 \mathrm{AG}-0.81 \mathrm{AL}+1.18 \mathrm{DTC}-0.61 \mathrm{SP}-1.98 \mathrm{FC}$.

A simplified DD-CSI model was developed as well by eliminating the variables with large $p$-values including crash time, road class, road character, road surface type, and road spatial feature. The following is the simplified DD-CSI model (Eq. (4)) and the variable coefficients are listed in Table 7:

simplified DD - CSI model : DD - CSI $=\frac{\exp \left[g_{4}(\boldsymbol{x})\right]}{1+\exp \left[g_{4}(\boldsymbol{x})\right]}$

where $\quad g_{4}(\boldsymbol{x})=4.88+0.63 \mathrm{LC}-0.81 \mathrm{VT}-0.58 \mathrm{LN}-0.87 \mathrm{SL}-1.77 \mathrm{AI}$ $-2.63 \mathrm{NTC}-0.70 \mathrm{FL}+0.73 \mathrm{ST}+0.33 \mathrm{AG}-0.85 \mathrm{AL}+1.08 \mathrm{DTC}-0.52 \mathrm{SP}$ $-2.01 \mathrm{FC}$
Table 6

Variables and coefficients for the comprehensive DD-CSI model

\begin{tabular}{|c|c|c|c|c|}
\hline Variable & Coeff. & Standard error & Wald chi-square & $p$-Valu \\
\hline Constant & 5.25 & 2.33 & 5.07 & 0.024 \\
\hline Crash time (CT) & 0.03 & 0.24 & 0.01 & 0.917 \\
\hline Light condition (LC) & 0.51 & 0.32 & 2.48 & 0.116 \\
\hline Vehicle type (VT) & -0.80 & 0.39 & 4.13 & 0.042 \\
\hline Road class (RC) & -0.59 & 0.57 & 1.07 & 0.301 \\
\hline Road character $(\mathrm{RCH})$ & 0.16 & 0.17 & 0.84 & 0.359 \\
\hline No. of lanes (LN) & -0.70 & 0.25 & 8.02 & 0.005 \\
\hline Speed limit (SL) & -0.84 & 0.26 & 10.65 & 0.001 \\
\hline Surface type (SUR) & 0.40 & 0.45 & 0.79 & 0.375 \\
\hline Special feature (SF) & -0.37 & 0.51 & 0.53 & 0.465 \\
\hline Area information (AI) & -1.69 & 0.67 & 6.36 & 0.012 \\
\hline $\begin{array}{l}\text { None/inoperative traffic } \\
\text { control (NTC) }\end{array}$ & -2.52 & 1.13 & 4.94 & 0.026 \\
\hline Flagger (FL) & -0.82 & 0.72 & 1.31 & 0.252 \\
\hline Stop sign/signal (ST) & 0.78 & 0.73 & 1.15 & 0.284 \\
\hline Age (AG) & 0.32 & 0.10 & 10.24 & 0.001 \\
\hline $\begin{array}{l}\text { Alcohol/drug } \\
\text { impairment (AL) }\end{array}$ & -0.81 & 0.67 & 1.45 & 0.228 \\
\hline $\begin{array}{l}\text { Disregarded traffic } \\
\text { control (DTC) }\end{array}$ & 1.18 & 0.57 & 4.30 & 0.038 \\
\hline $\begin{array}{l}\text { Speeding/too fast for } \\
\text { condition (SP) }\end{array}$ & -0.61 & 0.52 & 1.35 & 0.244 \\
\hline Following too close (FC) & -1.98 & 1.07 & 3.39 & 0.066 \\
\hline
\end{tabular}

$\mathrm{AIC}=244.0 ; \mathrm{SC}=316.4 ;-2 \log$ likelihood $=206.0$. Testing global null hypothesis: $\beta=0$ likelihood ratio chi-square (chi-square value, $p$-value): $128.9,<0.001$; score chisquare (chi-square value, $p$-value): $105.8,<0.001$; Wald chi-square (chi-square value, p-value): $58.9,<0.001$

Table 7

Variables and coefficients for the simplified DD-CSI mode

\begin{tabular}{lrlcr}
\hline Variable & Coeff. & Standard error & Wald chi-square & $p$-Value \\
\hline Constant & 4.88 & 1.80 & 7.32 & 0.007 \\
Light condition (LC) & 0.63 & 0.22 & 7.93 & 0.005 \\
Vehicle type (VT) & -0.81 & 0.39 & 4.22 & 0.040 \\
No. of lanes (LN) & -0.58 & 0.16 & 13.44 & $<0.001$ \\
Speed limit (SL) & -0.87 & 0.25 & 12.46 & $<0.001$ \\
Area information (AI) & -1.77 & 0.65 & 7.33 & 0.007 \\
None/inoperative traffic & -2.63 & 1.13 & 5.47 & 0.019 \\
control (NTC) & & & & 0.313 \\
Flagger (FL) & -0.70 & 0.70 & 1.02 & 0.291 \\
Stop sign/signal (ST) & 0.73 & 0.69 & 1.12 & 0.001 \\
Age (AG) & 0.33 & 0.10 & 11.12 & 0.199 \\
$\begin{array}{l}\text { Alcohol/drug } \\
\text { impairment (AL) }\end{array}$ & -0.85 & 0.67 & 1.65 & 0.049 \\
$\begin{array}{l}\text { Disregarded traffic } \\
\text { control (DTC) }\end{array}$ & 1.08 & 0.55 & 3.88 & \\
Speeding/too fast for & -0.52 & 0.49 & 1.12 & 0.289 \\
condition (SP) & & & & \\
Following too close (FC) & -2.01 & 1.06 & 3.57 &
\end{tabular}

AIC $=236.9 ; \quad S C=290.2 ;-2 \log$ likelihood $=208.9$. Testing global null hypothesis: $\beta=0$ likelihood ratio chi-square (chi-square value, $p$-value): $120.9,<0.001$; score chi-square (chi-square value, $p$-value): 103.6, <0.001; Wald chi-square (chi-square value, $p$-value): $60.5,<0.001$.

Table 8

Prediction accuracies of the CSI models

\begin{tabular}{lllll}
\hline \multirow{2}{*}{ Model } & \multicolumn{2}{l}{ Accuracy } & & $\sum(\text { ACS }- \text { CSI })^{2 a}$ \\
\cline { 2 - 4 } & Fatal (\%) & Injury (\%) & Total (\%) & \\
\hline Comprehensive DI-CSI & 28 & 95 & 92 & 22.3 \\
Simplified DI-CSI & 33 & 95 & 92 & 21.8 \\
Comprehensive DD-CSI & 22 & 95 & 91 & 22.9 \\
Simplified DD-CSI & 28 & 95 & 92 & 21.6 \\
\hline
\end{tabular}

a Sum of squared errors, where ACS = actual crash severity ( 1 for fatal and 0 for injury), and CSI = estimated crash severity index. 


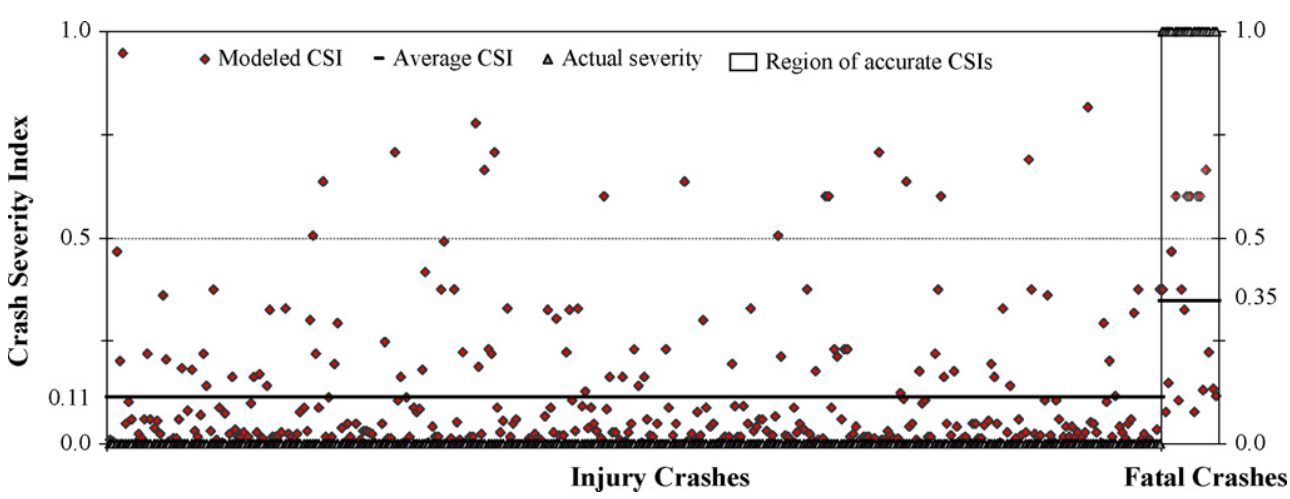

Fig. 2. CSIs estimated by the simplified DI-CSI model.

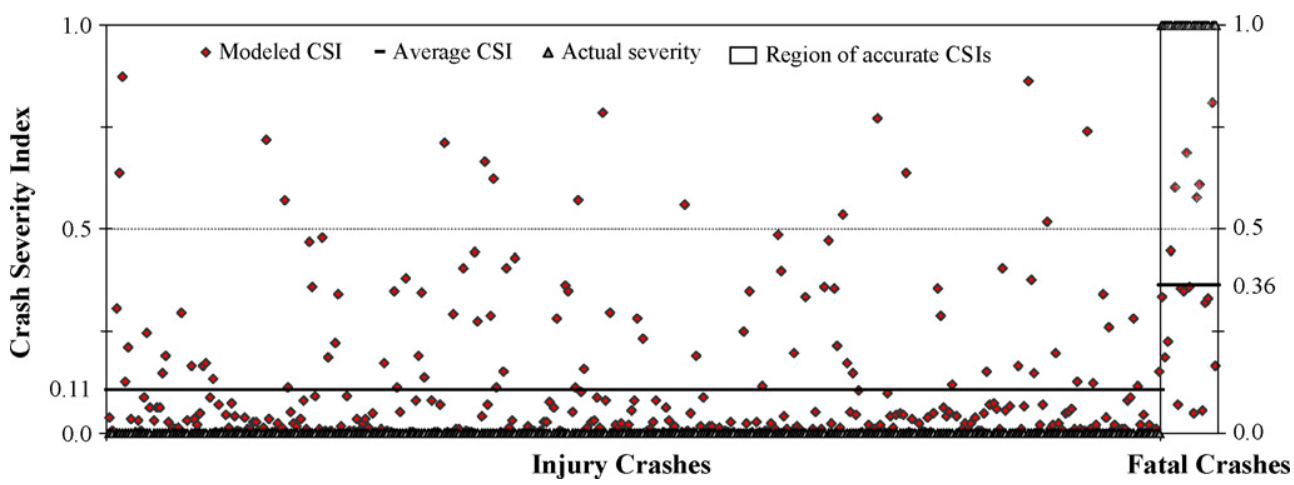

Fig. 3. CSIs estimated by the simplified DD-CSI model.

\subsection{Model validation}

The developed models were validated using 355 severe crash cases including 18 fatal crashes and 337 injury crashes in Kansas highway work zones in 2004. During the validation, researchers specified a CSI of one as a fatal crash and a CSI of zero as an injury crash. A CSI was calculated for each crash case based on the given crash variables. An estimated CSI number that is close to one indicated a very high risk level or a great likelihood of having a fatal crash for the given work zone travel conditions, while a CSI that is close to zero indicated a relative moderate risk level or a great likelihood of having a less severe crash such as an injury crash. The predicted CSI values were compared with the actual crash outcomes to illustrate the prediction accuracies. In addition, the four developed models were compared with each other.

Table 9

Example conditions with high CSIs

\begin{tabular}{|c|c|c|c|}
\hline \multirow[t]{2}{*}{ Crash variable } & \multicolumn{3}{|l|}{ High-CSI conditions } \\
\hline & No. 1 & No. 2 & No. 3 \\
\hline CSI & 0.62 & 0.75 & 0.88 \\
\hline Actual crash severity & Fatal & Fatal & Injury \\
\hline Age & 65 or older & $35-44$ & $35-44$ \\
\hline Crash time & 10:00 a.m. to $4: 00$ p.m. & 10:00 a.m. to 4:00 p.m. & $8: 00$ p.m. to $6: 00$ a.m. \\
\hline Light condition & Good condition & Good condition & Poor condition \\
\hline Vehicle type & Non-truck involved & Truck involved & Truck involved \\
\hline Road class & Other principal arterials and minor arterials & Other principal arterials and minor arterials & Other principal arterials and minor arterials \\
\hline Road character & Straight and level & Other alignments & Curved and level \\
\hline No. of lanes & 4 & 2 & 2 \\
\hline Speed limit & $51-60 \mathrm{mph}$ & $\geq 61 \mathrm{mph}$ & $\geq 61 \mathrm{mph}$ \\
\hline Surface type & Concrete & Blacktop & Blacktop \\
\hline Special feature ${ }^{a}$ & Impacted & Not present & Not present \\
\hline Area information & Urban area & Rural area & Rural area \\
\hline None/inoperative $\mathrm{TC}^{\mathrm{b}}$ & Not present & Not present & Not present \\
\hline Flagger/officer & Not present & Not present & Not present \\
\hline Stop sign/signal & Not present & Not present & Present \\
\hline Alcohol/drug impairment & Not present & Not present & Not present \\
\hline Disregarded TC & Present & Not present & Not present \\
\hline Speeding/too fast for condition & Not present & Not present & Present \\
\hline Following too closely & Not present & Not present & Not present \\
\hline
\end{tabular}

a Special features may include bridge, overhead bridge, railroad bridge, railroad crossing, interchange, ramp, and other.

b Traffic control. 
Table 8 presents the comparison results between the estimated CSI numbers and the real crash severities. It shows minor differenced between the two CSI models in each category in terms of accuracy. Figs. 2 and 3 graphically illustrate the estimated indices of the crashes using the two simplified models, respectively. When setting 0.5 as the criterion for the CSI (i.e., CSI $\geq 0.5$ for likelihood of having a fatal crash and CSI $<0.5$ for likelihood of having an injury crash), on average, the models predicted about five fatal crash cases (with CSI values greater than or equal to 0.5 ) out of the 18 fatal cases. On the other hand, all four models predicted about $95 \%$ of the injury cases $(\mathrm{CSI}<0.5)$. Based on the 2004 injury and fatal crash data, the simplified DI- and DD-CSI models were slightly better than the comprehensive models for both accuracies in percentage and average estimated CSI values.

According to these four models, the average CSI for the travel conditions of injury crashes were around 0.11 , while the average CSI for fatal cases fell between 0.3 and 0.36 (comprehensive DI-CSI model: 0.32; simplified DI-CSI model: 0.35; comprehensive DD-CSI model: 0.30; simplified DD-CSI model: 0.36). Generally, the models captured the differences of the input work zone travel conditions and successfully separated different traffic conditions by assigning them with different CSI values (i.e., not dramatically clustered in a certain small range). However, the accuracy of using CSI to predict the fatal crashes may be further improved through future research. For example, a larger dataset including sufficient fatal crash information may be used when available in future development.

Table 9 present some examples of work zone travel conditions with very high CSI values estimated by the comprehensive DD-CSI model. Typically, risk factors such as poor light condition, truck involvement, having only two travel lanes, and high speed limit may lead to high CSI values and equivalently, high risk levels. Note that, in the table, the travel conditions with very-high CSI values included an injury case. This indicated that a high CSI may not necessarily coincide with a fatal crash; a CSI with a high value implies that the condition is risky and it has a high likelihood of causing high-severity crashes such as fatal crashes.

\section{Conclusion and recommendation}

In this study, four CSI models were developed for risk level assessment in work zones based on crash severity modeling. The models incorporated the risk factors that were determined using chi-square tests, $\mathrm{CMH}$ statistics, and results of the previous crash characteristic study. The CSI models were designed to quantify the risk level of a work zone with a numerical value between zero and one. A CSI of one indicates a very high risk level in a given work zone, which infers that a fatal crash might take place if a crash occurs.

Two groups of models were developed, including two driverindependent CSI or DI-CSI models and two driver-dependent CSI or DD-CSI models. The DI-CSI models were developed for the work zone travel risk assessment without considering human factors or specific driving groups; the DD-CSI models, on the other hand, addressed the risks associated with travel conditions along with human errors and the characteristics of specific driving group. Thus, DD-CSI models are suitable for the driving risk assessment for given driving groups in given highway work zones.

Generally, the CSI models captured the differences between the work zone conditions with fatal and injury crashes. Model validation showed that the CSIs for most work zones with severe crashes were consistent with the actual crash severity outcomes. The researchers recommend that the CSI models should be used in work zone planning or work zone safety inspection so that work zone risk factors could be identified and safety countermeasures could be developed accordingly to mitigate risk. Utilization of CSI models will help engineers to reveal work zone risks that are created by subtle combinations of a wide range of variables which otherwise may be not detectable solely based on engineering experience. Model validation showed minor accuracy differences between the comprehensive models and the simplified models. Therefore, the researcher could not reach the conclusion on which models were credibly superior. Additional validations with large datasets are needed. When there is sufficient information, it is recommended that the comprehensive models be used since they include all risk factors identified based on both statistical tests and crash characteristic studies.

While the predicted CSI values for most of the travel conditions for injury crashes were consistent with the actual crash severity observed, the predicted CSI values for some of the fatal crash cases were not consistent with the actual severity outcomes. Reasons for these inconsistencies may include:

The covariate pattern examination showed that both fatal and injury crashes were observed for some work zone conditions. A covariate pattern is a certain combination of crash variables with certain values. This suggests that a minor fraction of fatal and injury crashes could not be separated by travel conditions shown in the KDOT crash reports. The CSI numbers for these risk conditions would be either biased to a low value (if the conditions were dominated by injury crashes) or to a high value (if the conditions were dominated by fatal crashes).

In both model development and model validation datasets, the existence of very severe injury crashes (e.g., near-fatal injury crashes) and some fatal crashes, whose fatalities were due to reasons other than work zone risk factors such as physical vulnerability or not wearing a seat belt, would reduce the accuracy of the models. Using more detailed crash severity classification may eliminate or mitigate this type of error.

The crash data used for model validation had only 18 fatal crash cases. The size of the fatal crash sample might not be large enough to validate the developed models under typical fatal conditions.

Future research is recommended for the improvement of the CSI models. When available, a larger dataset should be used for the future development and validation of the CSI models. The CSI models can also be improved by taking into consideration the crashes of other severities such as property-damage-only crashes. In addition, more detailed classification of crash severities should be used during future development of CSI models so that the CSIs with intermediate values can be interpreted with corresponding severities. Information on work zone configurations, if available, should also be included in the CSI models to improve their accuracies.

\section{Acknowledgements}

The authors would like to thank Mr. Anthony Alrobaire and Mr. Rex McCommon from KDOT for their valuable help and advice during the course of this study. Heartfelt thanks also go to Dr. Yaozhong $\mathrm{Hu}$ in the Department of Mathematics at the University of Kansas for his valuable advice during the course of this study. The cooperation and assistance from other KDOT staff during the process of crash data collection are also greatly appreciated.

\section{References}

Chang, H., Yeh, T., 2006. Risk factors to driver fatalities in single-vehicle crashes: comparisons between non-motorcycle drivers and motorcyclists. Journal of Transportation Engineering 132 (3), 227-236.

Chen, W., Jovanis, P.P., 2000. Method for identifying factors contributing to driverinjury severity in traffic crashes. Journal of the Transportation Research Board $1707,1-9$.

Chirsa-Chavala, T., Mak, K.K., 1986. Identification of accident factors on highway segments: a method and applications. Journal of the Transportation Research Board 1068, 52-58. 
Dissanayake, S., Lu, J., 2002. Analysis of severity of young driver crashes, sequential binary logistic regression modeling. Journal of the Transportation Research Board 1784, 108-114

Harrell Jr., F.E., 2001. Regression Modeling Strategies-with Application to Linear Models, Logistic Regression, and Survival Analysis. Springer-Verlag, New York Inc, pp. 215-221.

Hill, R.W., 2003. Master Thesis: Statistical Analysis of Fatal Traffic Accident Data. Texas Tech University, Lubbock, Texas.

Kim, K., Kim, S., Yamashita, E., 2000. Alcohol-impaired motorcycle crashes in Hawaii, 1986-1995. Journal of the Transportation Research Board 1704, 77-85.

Li, Y., 2007. Analyzing highway work zone crashes and traffic control effectiveness. Ph.D. Dissertation. The University of Kansas, Lawrence, Kansas.
Li, Y., Bai, Y., 2006. Investigating the characteristics of fatal crashes in the highway construction zones. In: CIB W99 International Conference on Global Unity for Safety \& Health in Construction, Beijing, China, June 28-30, pp. 301309.

Lu, G., Noyce, D.A., McKendry, R.J., 2006. Analysis of the magnitude and predictability of median crossover crashes utilizing logistic regression. In: Proceedings of the TRB 85th Annual Meeting, CD-ROM, January 22-26.

Ouyang, Y., Shankar, V., Yamamoto, T., 2002. Modeling the simultaneity in injury causation in multi-vehicle collisions. Journal of the Transportation Research Board $1704,143-152$.

SAS Help and Documentation (SAS), 2003. The Logistic Procedure-Model Fitting Information. SAS Institute Inc., Cary, NC. 